\title{
Improving motor function after chronic stroke by interactive gaming with a redesigned MR-compatible hand training device
}

\author{
LOUKAS G. ASTRAKAS ${ }^{1}$, GIANLUCA DE NOVI ${ }^{2,3}$, MARK P. OTTENSMEYER $^{2,3}$, \\ CHRISTIAN PUSATERE ${ }^{4,5}$, SHASHA LI ${ }^{3-5}$, MICHAEL A. MOSKOWITZ $^{5-7}$ and A. ARIA TZIKA ${ }^{4,5,8}$ \\ ${ }^{1}$ Medical Physics Laboratory, Faculty of Medicine, University of Ioannina, Ioannina 45110, Greece; \\ ${ }^{2}$ Medical Device and Simulation Laboratory, Department of Radiology, Massachusetts General Hospital, Boston, MA 02114; \\ ${ }^{3}$ Department of Radiology, Harvard Medical School, Boston, MA 02115; ${ }^{4}$ Nuclear Magnetic Resonance Surgical Laboratory, \\ Department of Surgery, Center for Surgery, Innovation and Bioengineering, Massachusetts General Hospital, \\ Boston, MA 02114; ${ }^{5}$ Athinoula A. Martinos Center of Biomedical Imaging, Charlestown, MA 02129; \\ ${ }^{6}$ Department of Neurology, Neuroscience Center, Massachusetts General Hospital, Boston, MA 02114; \\ Departments of ${ }^{7}$ Neurology and ${ }^{8}$ Surgery, Harvard Medical School, Boston, MA 02115, USA
}

Received September 8, 2020; Accepted December 4, 2020

DOI: $10.3892 / e t m .2021 .9676$

\begin{abstract}
New rehabilitation strategies enabled by technological developments are challenging the prevailing concept of there being a limited window for functional recovery after stroke. In this study, we examined the utility of a robot-assisted therapy used in combination with a serious game as a rehabilitation and motor assessment tool in patients with chronic stroke. We evaluated 928 game rounds from 386 training sessions of 8 patients who had suffered an ischemic stroke affecting middle cerebral artery territory that incurred at least 6 months prior. Motor function was assessed with clinical motor scales, including the Fugl-Meyer upper extremity (FM UE) scale, Action Research Arm Test, Modified Ashworth scale and the Box and Blocks test. Robotic device output measures (mean force, force-position correlation) and serious game score elements (collisions, rewards and total score) were calculated. A total of 2 patients exhibited a marginal improvement after a 10-week training protocol according to the FM UE scale and an additional patient exhibited a significant improvement according to Box and Blocks test. Motor scales showed strong associations of robotic device parameters and game metrics
\end{abstract}

Correspondence to: Dr A. Aria Tzika, Nuclear Magnetic Resonance Surgical Laboratory, Department of Surgery, Center for Surgery, Innovation and Bioengineering, Massachusetts General Hospital, 51 Blossom Street, Room 261, Boston, MA 02114, USA

E-mail: atzika@hms.harvard.edu

Abbreviations: ARAT, Action Research Arm Test; FM UE, Fugl-Meyer upper extremity; MR, magnetic resonance; MR_CHIROD, MR compatible Hand-Induced RObotic Device; XML, Extensible Markup Language

Key words: stroke, hand, rehabilitation, robotics with clinical motor scale scores, with the strongest correlations observed for the mean force $(0.677<\mathrm{P}<0.869)$, followed by the number of collisions $(-0.670<\mathrm{P}<-0.585)$. Linear regression analysis showed that these indices were independent predictors of motor scale scores. In conclusion, a robotic device linked to a serious game can be used by patients with chronic stroke and induce at least some clinical improvements in motor performance. Robotic device output parameters and game score elements associate strongly with clinical motor scales and have the potential to be used as predictors in models of rehabilitation progress.

\section{Introduction}

Stroke remains a major cause of morbidity and disability (1). In the USA, stroke is the most frequent cause of adult-onset disability and the cost of care for stroke patients is among the fastest-growing expenses for Medicare (2). Therapy can improve recovery and reduce long-term disability in patients who have suffered a stroke (3). Post-stroke rehabilitation services are generally targeted to the initial post-acute phase, predominantly the first several weeks or, perhaps up to a few months. This limited period of intervention derives from the perception of deficit permanence after a 3-6-month 'critical window' of enhanced neuroplasticity after stroke (4), and thus, expectations of no subsequent improvement. Remarkably, many studies have demonstrated a gradient of rehabilitation therapy responsiveness in stroke patients that extends beyond a year (5). New rehabilitation strategies enabled by new technological developments have been shown to yield performance gains in chronic stroke patients $(6)$. Serious games $(7,8)$ and robotic devices (9) have emerged as promising rehabilitation tools for the improvement of motor function and quality of life.

Serious game-based interventions immerse patients in enriched, stimulating computer simulated environments in which they interact with virtual objects while performing functional task-specific activities. Real-time feedback, 
predetermined task goals, and avatars are used to increase a patient's motivation and engagement. The amenability of such gaming interventions to being accessed via commercially available game alleviates cost while maximizing convenience in that rehabilitation protocols can be followed at home. Several studies have shown modest advantages of virtual reality-based interventions compared to traditional repetitive task practice protocols (10).

Clinical studies have shown that robot-assisted therapy can enhance neurological recovery (11). The main advantage of rehabilitation robots is that they can deliver high-dosage, high-intensity training, which is especially beneficial for patients working to improve motor system dysfunction due to stroke or spinal cord disease. Incremental improvements in clinically observed performance following intensive robotic therapy, although small, are statistically significant and promising for a variety of patient population, including chronic stroke patients (12).

The aim of the present study was to examine whether and to what extent the motor performance of chronic stroke patients can be improved with a combination of a robot-assisted therapy and serious gaming. Additionally, we assessed the potential utility of robotic device output parameters and game performance metrics as indirect indices of motor improvement.

\section{Materials and methods}

Subjects. The study cohort included eight stroke patients (5 women and 3 men) ranging in age from 39 to 60 years old who were right-handed according to the Edinburgh Handedness Inventory (13). They were recruited through the registries of stroke survivors who agreed to be contacted for stroke recovery studies that are maintained at Massachusetts General Hospital. Each had suffered an ischemic stroke affecting the left middle cerebral artery territory at least 6 months prior to recruitment. According to their medical records, they presented with acute unilateral loss of hand strength (Medical Research Council scale score $<4$, on $0-5$ scale in which 5 is normal) that lasted for $>48 \mathrm{~h}$. They did not have hearing, vision, language, or cognitive deficits. Institutional review board approval of the study was granted by the Massachusetts General Hospital Human Research Committee (protocol number 2005P000570) and all participants provided written informed consent.

Data collection. We evaluated a total of 928 game rounds distributed over 386 training sessions. Patients were trained with a robotic hand rehabilitation system coupled to an interactive game for 45 min per day, 3 days per week, over a 10 -week period. Each training session consisted of four 8-minute-long scenarios separated by 1 - to 5 -minute rest breaks. One session was conducted on each of three training days per week. Training was conducted at the patients' homes under supervision to ensure compliance. Motor performance was assessed prior to rehabilitation training (Pre), after finishing the treatment period (Post), and again 1 month after finishing the treatment in a follow-up assessment (FU) intended to probe the persistence of motor benefits over time. Motor performance was assessed via four clinical motor scales: The Fugl-Meyer scale, which we used to assess sensorimotor impairment (includes upper extremity, wrist, hand, coordination, sensation, passive joint motion, joint pain, and total scores) (14); the Action Research Arm Test (ARAT), which includes grasp, grip, pinch, gross movement, and total scores (15); the Modified Ashworth scale to assess spasticity of the elbow, wrist, fingers, and thumb (16); and the Box and Blocks test of gross manual dexterity (17).

Rehabilitation system. Patients underwent training with a newly re-designed robotic hand rehabilitation device called the Magnetic Resonance compatible Hand-Induced RObotic Device, version 3 (MR_CHIROD v3) (18-20). It was engineered to provide adjustable levels of force for handgrip exercising (Fig. 1). Continuous, grip-opposing, restoring force was provided by a low-friction glass cylinder/graphite piston assembly under the control of an electronic pneumatic pressure regulator with a portable air compressor that allowed the MR_CHIROD v3 to be used in a home environment. The MR_CHIROD v3 device was linked to a low-cost, Arduino-compatible microcontroller via a simple USB serial interface (57.6-kbps data transfer rate) thereby allowing pressure control, force/displacement acquisition, and interface with a laptop computer.

Game software. A serious game was developed in Microsoft Visual C++ for use on a Microsoft Surface (Microsoft Corp., Redmond, WA). The user plays the game via an avatar, namely a small green alien in a flying saucer that travels through a linear scrolling labyrinth (right to left scrolling). The speed of the saucer starts slow and increases linearly to a final level for each stage. The saucer flies through a scene, earning points for collision-free flight. The scene is populated with obstacles and rewards, collisions with which contribute negatively and positively to the user's score, respectively. During the game, the patient uses the MR_CHIROD handle to control the altitude of the saucer such that the handle position controls saucer height on the screen directly, with the fully open and fully closed positions corresponding to the lowest and highest screen positions. The goal of the game is to maximize one's overall score by avoiding obstacles and collecting rewards (Fig. 2). To increase user involvement, a soundtrack is looped and several sound effects are played upon collision with obstacles and rewards.

The game scenario was parametrized through an Extensible Markup Language (XML) script ('stage script') that specifies what the game engine loads in the form of sprites (graphic elements such as the avatar, obstacles, and rewards), the background picture, and game information. The stage script specifies obstacle and reward point values as well as all game components and mechanisms, including speed, sounds, and supportive visuals. Obstacles can be avoided with large, low accuracy motions to reach a safe position, whereas reward contact requires fine position control within a small range of motion. However, for a greater challenge in high-level play, users face more closely-spaced obstacles that leave narrow passages that require fine control to negotiate. The combination of obstacles and rewards allows the design of precise and complex trajectories for training gross motor and fine grip motions.

Prior to the commencement of each stage of the game, the prescribed pressure command is sent to the MR_CHIROD v3. When the game starts, the game engine polls the MR_CHIROD 


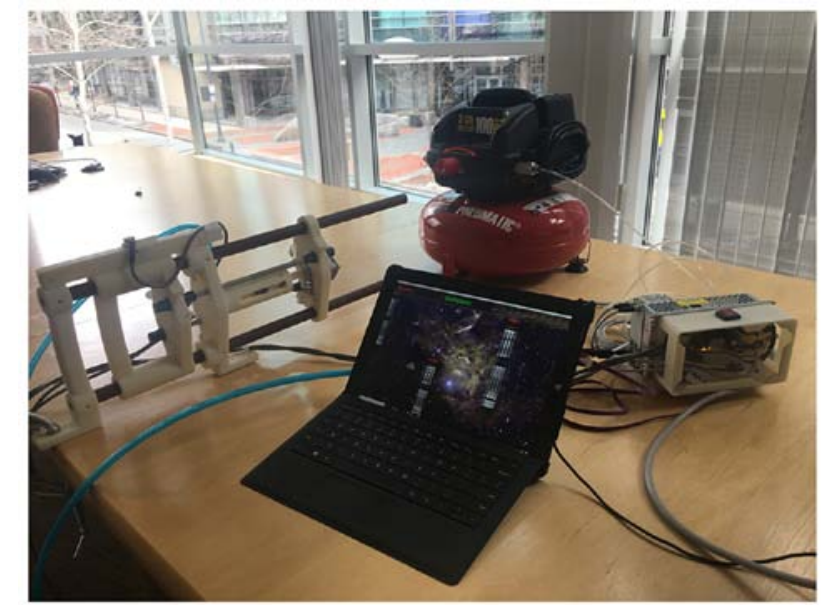

Figure 1. Magnetic resonance compatible Hand-Induced RObotic Device v3. Robotic hand rehabilitation system.

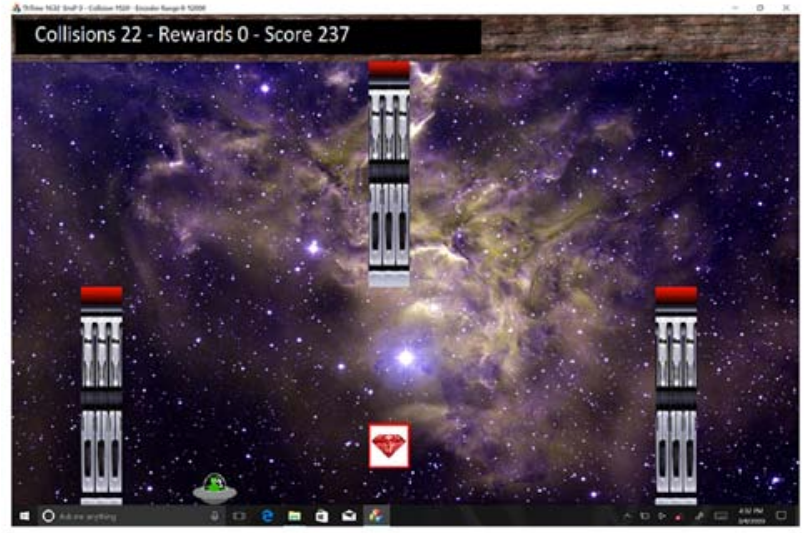

Figure 2. Screenshot view of the serious rehabilitative game showing the player's avatar (alien in a spaceship), three obstacles (columns with red tops), and a reward (red gem).

interface to retrieve the handle position in encoder counts and normalizes the position based on the range of motion set by end-stops on the device appropriate to each patient, and then updates the vertical and horizontal position coordinates of the avatar.

Game scores are calculated continuously based on the following formula:

$$
\mathrm{S}(\mathrm{t})=\mathrm{S}(\mathrm{t}-1)+\mathrm{R}(\mathrm{t})-\mathrm{O}(\mathrm{t})+\mathrm{g}
$$

where the instantaneous score $\mathrm{S}(\mathrm{t})$ is equal to the previous score $\mathrm{S}(\mathrm{t}-1)$ plus any reward collected, $\mathrm{R}(\mathrm{t})$, minus any penalty for hitting an obstacle, $\mathrm{O}(\mathrm{t})$, plus the constant $\mathrm{g}$, which is the incremental score for each time interval. The scoring algorithm was tuned by adjusting $\mathrm{R}, \mathrm{O}$, and $\mathrm{g}$ in the stage script. The game was programmed to save each user's position trajectory, events at each time interval, score, handle pressure, and timestamp into a secondary XML script, which we used for offline analysis of raw and processed information. The stage script was individualized such that the handle force setting was set to $\sim 75 \%$ of the user's maximal grip strength, thereby requiring our patients to exert effort and generate correspondingly large motor cortex activation, while not being so high as to impede completion of the training sessions.

Data analysis. For the purposes of the present study analyses, motor impairment was classified according to a three-level Fugl-Meyer upper extremity (FM UE) scale scheme (21), in which an FM UE score <20 was classified as severe, a score in the range of 20-40 was classified as moderate, and a score $>40$ was classified as mild. Improvement of at least 4.25 on the FM UE scale (22) and/or a smallest real difference of at least 5.5 blocks/min on the Box and Blocks test (23) were considered signs of clinically meaningful improvement in grip ability.

The mean force and the Pearson correlation coefficient between handle position and force (an indirect measure of grip control) were calculated for each round. These device metrics and the game metrics (final score, collisions, and rewards) for each of four scenarios were averaged for each session. Associations of device and game metrics with clinical motor scale scores were assessed with the Spearman @ correlation coefficient after Bonferroni correction of multiple comparisons. Stepwise linear regression analysis was used to assess which metrics were significant predictors of motor performance, as indexed by FM UE, ARAT grip, and Box and Blocks scores. All statistical analyses were performed in SPSS version 23.0 (IBM, Inc.). Analyses with two-tailed P-values $<0.05$ were regarded as statistically significant.

\section{Results}

Log files from a total of 928 game rounds were processed offline. Of 389 training sessions, 6 early data sets were discarded due to equipment malfunction, affecting 2 of the 8 patients' results. All 8 patients had significant motor impairments before they began training (Table I). Specifically, according to our three-level FM UE scale-based classification scheme, two patients $(\# 4, \# 5)$ had severe (score $<20)$ impairment while the other four had moderate (score, 20-40) motor impairment prior to starting the training program. ARAT grip and Box and Blocks scale scores also indicated that patients \#4 and \#5 had severe motor impairment (Table I). Patient \#5 presented with spasticity (Modified Ashworth scale scores: Elbow, 3; wrist, 4; fingers, 3; and thumb, 3). Patients \#1 and \#5 showed marginal progress during the rehabilitation period, as indicated by attending a clinically important difference for grip ability of $\geq 4.25$ on the FM UE scale. According to the criterion of a smallest real difference of 5.5 blocks $/ \mathrm{min}$ in the Box and Blocks test, patient \#3 showed significant improvement.

In most cases, the evolution of robotic device metrics (Fig. 3) and game metrics (Fig. 4) followed discernable patterns. For example, the mean forces exerted by subjects \#2, \#3, \#7 and \#8 were higher than those of the other patients and remained at similar levels throughout rehabilitation (Fig. 3), corresponding with the FM UE assessments of their motor functions. The same subjects had the highest force-position correlations. Subject \#1 showed a discernable increase, nearly reaching the same correlation value by the end of training (Fig. 3). Regarding game performance, the subjects could be divided into three types: i) good performers (\#1, \#2 and \#3), with relatively high scores, a large number of rewards, and few 
Table I. Demographic characteristics of and motor assessment scores for each patient.

\begin{tabular}{|c|c|c|c|c|c|c|c|c|c|c|c|}
\hline \multirow[b]{2}{*}{ Patient no. } & \multirow[b]{2}{*}{ Age, years } & \multirow[b]{2}{*}{ Gender } & \multicolumn{3}{|c|}{ FM UE } & \multicolumn{3}{|c|}{ ARAT grip } & \multicolumn{3}{|c|}{ Box and Blocks } \\
\hline & & & Pre & Post & $\mathrm{FU}$ & Pre & Post & $\mathrm{FU}$ & Pre & Post & $\mathrm{FU}$ \\
\hline 1 & 39 & Female & 27 & 31 & 32 & 12 & 12 & 12 & 43 & 45 & 47 \\
\hline 2 & 64 & Male & 36 & 36 & 36 & 12 & 12 & 12 & 54 & 56 & 57 \\
\hline 3 & 50 & Male & 36 & 36 & 36 & 12 & 12 & 12 & 69 & 82 & 92 \\
\hline 4 & 59 & Female & 18 & 21 & 21 & 0 & 0 & 0 & 0 & 0 & 2 \\
\hline 5 & 60 & Female & 15 & 21 & 17 & 3 & 6 & 6 & 0 & 0 & 0 \\
\hline 6 & 46 & Female & 28 & 29 & 28 & 12 & 12 & 12 & 13 & 13 & 14 \\
\hline 7 & 40 & Male & 32 & 32 & 32 & 12 & 12 & 12 & 46 & 44 & 45 \\
\hline 8 & 33 & Female & 32 & 3 & 32 & 12 & 12 & 12 & 51 & 56 & 56 \\
\hline
\end{tabular}

Pre, before training; Post, at the conclusion of training; FU, at the follow-up test 1 month after completing the training; FM UE, Fugl-Meyer upper extremity; ARAT, Action Research Arm Test.
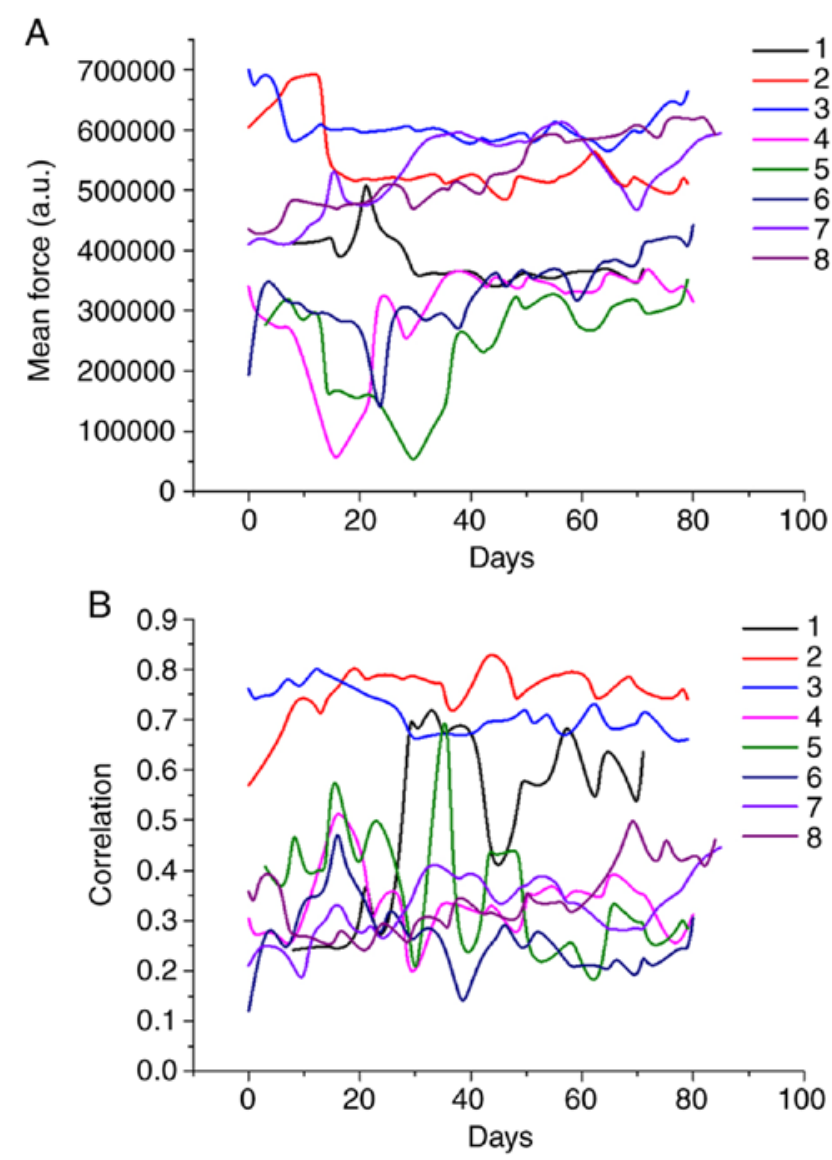

Figure 3. Robotic metrics. (A) Evolution of mean force and (B) position-force correlation during training for each subject. Numbers 1-8 correspond with patient numbers. a.u., arbitrary units.

collisions, albeit with limited improvement; ii) patients with (at least initially) low scores who achieved marked improvement (\#4, \#5, \#6, and \#7); and iii) one poor performer (\#8) whose scores started and remained low. The middle group patients tended to show rapid improvement initially followed by a gradual improvement such that their metrics approached the levels of the first group.
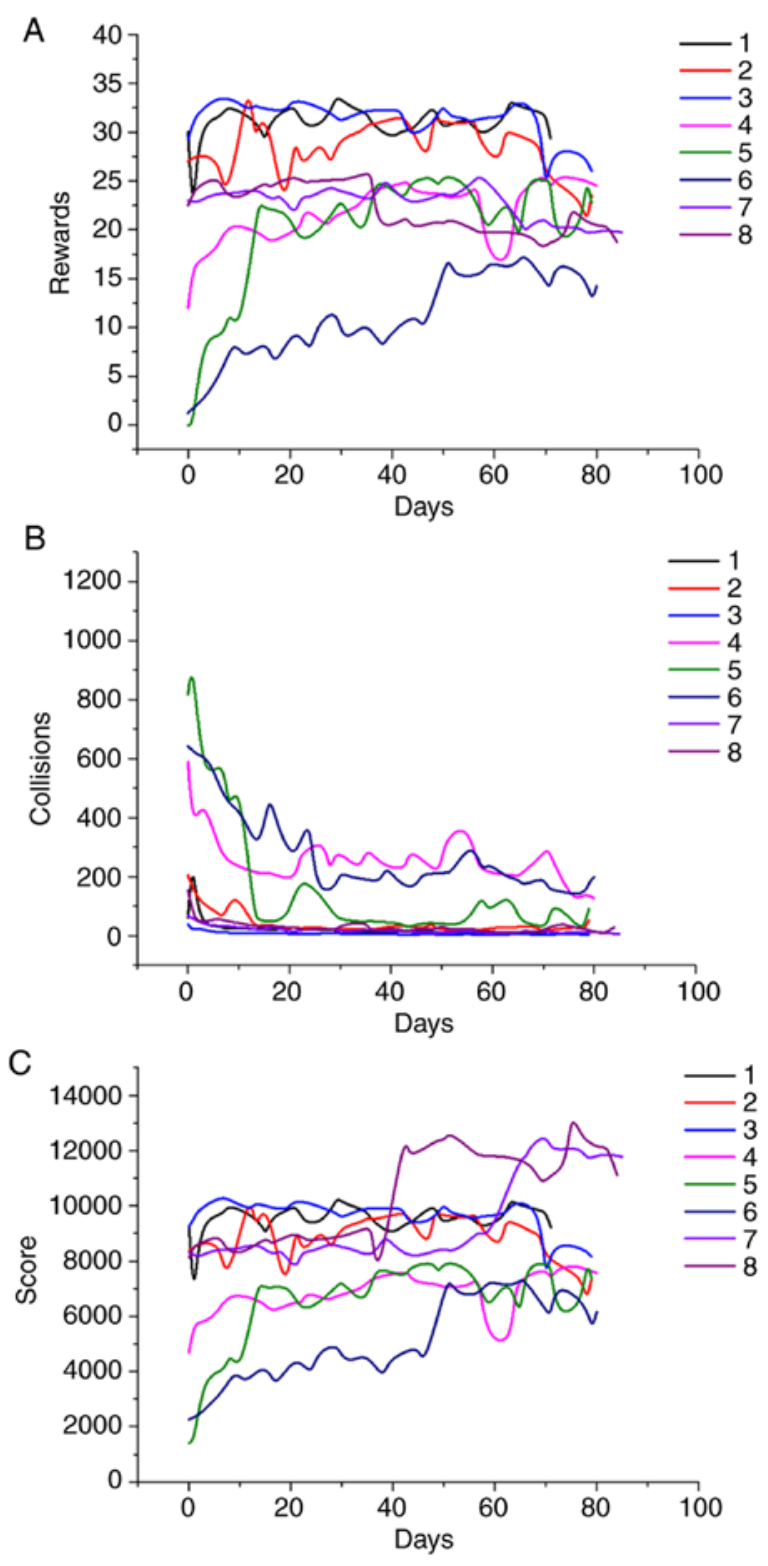

Figure 4. Changes in game metrics over the training period, including (A) rewards, (B) collisions and (C) final scores. Numbers 1-8 correspond with patient numbers. 
Table II. Analysis of robotic system and game metrics correlations with motor assessment scale scores.

\begin{tabular}{|c|c|c|c|c|c|c|}
\hline \multirow[b]{2}{*}{ Parameter } & \multicolumn{2}{|c|}{ FM UE } & \multicolumn{2}{|c|}{ ARAT grip } & \multicolumn{2}{|c|}{ Box and Blocks } \\
\hline & $\varrho$ & P-value & $\varrho$ & P-value & $\mathrm{P}$ & P-value \\
\hline \multicolumn{7}{|l|}{ Robotic system } \\
\hline Mean force & 0.869 & $<0.001$ & 0.677 & $<0.001$ & 0.865 & $<0.001$ \\
\hline Position-force correlation & 0.651 & $<0.001$ & 0.228 & 0.234 & 0.613 & $<0.001$ \\
\hline \multicolumn{7}{|l|}{ Game } \\
\hline Rewards & 0.528 & 0.002 & 0.330 & 0.070 & 0.488 & 0.005 \\
\hline Collisions & -0.642 & $<0.001$ & -0.585 & 0.001 & -0.670 & $<0.001$ \\
\hline Score & 0.590 & $<0.001$ & 0.602 & $<0.001$ & 0.636 & $<0.001$ \\
\hline
\end{tabular}

FM UE, Fugl-Meyer upper extremity; ARAT, Action Research Arm Test.

We observed strong correlations of robotic device metrics and game metrics with motor ability scale indices (Table II). Of the five device and game metrics included in our correlational analyses, the robotic device metric mean force had the strongest correlations with motor ability scale indices. Among the three game metrics, number of collisions had the strongest correlation with motor scale scores. Stepwise linear regression analysis indicated that device and game metrics were indeed predictive of motor scale scores. Specifically, independent predictors of FM UE score were mean force (standardized Beta $=0.662 ; \mathrm{P}<0.001$ ) and collisions (standardized Beta $=-0.273 ; \mathrm{P}<0.039$ ). Meanwhile, independent predictors of ARAT grip score were mean force (standardized Beta $=0.336$; $\mathrm{P}=0.049$ ) and collisions (standardized Beta=-0.471; $\mathrm{P}=0.007$ ). Finally, independent predictors of Box and Blocks scores were mean force (standardized Beta $=0.585 ; \mathrm{P}<0.001$ ), collisions (standardized Beta $=-0.289 ; \mathrm{P}=0.007$ ), and correlation (standardized Beta $=0.223 ; \mathrm{P}=0.033$ ). In order of most to least predictable, Box and Blocks scale $\left(\mathrm{R}^{2}=0.821\right)$, FM UE scale $\left(\mathrm{R}^{2}=0.700\right)$, and ARAT grip scale $\left(\mathrm{R}^{2}=0.499\right)$ scores all correlated very significantly with metrics (all $3, \mathrm{P}<0.001$ ).

\section{Discussion}

In this study, we examined the utility of a hand rehabilitation system for stroke patients consisting of a serious game coupled to a magnetic resonance (MR)-compatible robotic device. Combining robotic technology with serious gaming has the potential to achieve good patient engagement, immersion, and motivation and thus to produce motor skill-relearning associated neuronal activation that can, in principle, promote neuroplasticity (24). We observed large game metric improvements in the early days of training, suggesting that more measurements should be monitored over the initial weeks of training to establish stronger conclusions. These preliminary results hint that finer-grained temporal analyses will show that such data would be helpful for building a motor ability improvement prediction model.

Very few studies $(25,26)$ have attempted to assess the additional benefits of this combination of technologies. Patients \#1 and \#5 in our study showed marginal motor improvements on the FM UE scale $(27,28)$. Compared with conventional therapy, these improvements appear to be similar to or greater than is achieved with conventional therapy (28). Nevertheless, the combination of robotics with serious gaming addresses known limitations (29) of conventional post-stroke rehabilitation including limited availability of specialized facilities, transportation challenges, patient non-compliance, and costs.

Previous attempts to predict motor outcomes have been hindered by methodological flaws and a lack of external validation $(30,31)$. The inclusion of neuroimaging and neurophysiological indices in clinical outcome predictive models could improve their accuracy $(30,31)$. These additional data become more important when clinical status is determined based on self-reported assessments, a common practice for chronic stroke patients who often follow prescribed, but unsupervised, rehabilitation protocols at home. Self-reported clinical monitoring becomes even less reliable when patients have cognitive deficits. The strong correlations of our device and game metrics with motor scale scores suggests that our hand rehabilitation system can provide indirect but objective assessments of motor improvement. Moreover, MR compatibility allows state of the art neuroimaging during game play to provide insights into underlying structural remodeling and reorganization processes of functional recovery in the brain during the performance of rehabilitation tasks.

Although gaming in post-stroke rehabilitation has been considered a promising therapeutic resource, there has been limited examination of clinical efficacy. Therapeutic outcomes were evaluated in only about half of 31 reviewed studies and no relationship was found between game score and clinical findings in the remaining studies (32). Even so, correlation between game score and clinical tests have the potential to guide the development of biomedical systems that aid in treatment and evaluation (32). In our game, the score element 'number of collisions' had stronger associations with clinical motor scale scores than 'number of rewards' or 'total score'. Hence, our results suggest that game metrics could be used for clinical evaluation, but also that different weighting of score elements would be appropriate indicated.

Regarding gender, our sample was too small to allow any meaningful analysis of gender differences. It is evident that, in our sample, the three male patients started the rehabilitation period with generally better clinical scores, and thus less 
severe stroke-related impairment, than the five female patients; the highest FM UE pre-rehabilitation period score observed among the woman was the same as the lowest observed among the men. Both patients that showed marginal improvement, according to FM UE scores, were women. Thus, it may be that our protocol was particularly beneficial to patients with relatively more severe impairment.

Although the introduction of computer gaming into rehabilitation is quite new, robotics have been used extensively for motor performance assessments during rehabilitation (33). For example, the InMotion2 robot (Bionik Laboratories, Corp., Toronto, Canada), a commercial version of the MIT-Manus, provides automated estimates upper extremity clinical scores for chronic stroke patients based on multiple regression models of kinetic and kinematic macro-metrics (34). In agreement with this approach, we observed strong correlations between robotic device metrics and clinical scores. Importantly, our stepwise regression modeling demonstrated that the inclusion of gaming metrics increased the predictive power of clinical score models.

System advantages. Notably, the MR_CHIROD v3 system was designed with features that facilitate its use and dissemination. The system's USB serial connectivity and available WiFi compatibility allow it to be used with multiple commercial operating systems and game consoles. The master-side of the polling-based serial communication protocol can also be implemented in different programs. To enable ease of replication and wider use of the system, it has multiple 3D-printable and uncomplicated machinable components as well as low-cost electronic and pneumatic components.

The strong magnetic fields characteristic of the modern MR machines limit the range of actuators, sensors, and materials that can be used in an MR-compatible rehabilitation devices. Ferrous materials, electromagnetic actuators, and unshielded conductors maybe forbidden for use in MR scanners altogether for safety reasons or poorly suited due to imaging data distortion. The MR-compatibility of the MR_CHIROD v3 robotic device-made possible by plastic parts (3D-printed and machined), shielded sensors/cables, and MR-compatible ball bearings and other components-mean that patients brain activation can be analyzed during its use. Generally, upper-extremity rehabilitation devices are not made to MR-compatible (35); for exceptions see (36). In addition to being MR-compatible, the currently used MR_CHIROD version 3 (manuscript describing the design and testing is in preparation) is portable and suitable for standardized use across clinical and home environments (18). Whereas the high cost of fabricating our prior design of the MR_CHIROD device (version 2) was limiting (19), the version 3 redesign yielded fabrication cost reduction as well as overall simplification of the fabrication process.

Finally, it is our view that the combination of robotic technologies with serious gaming can be applied as a rehabilitation tool as well as in multiparametric modeling of motor performance and/or of the rehabilitation outcome. Whereas device metrics are strongly linked to motor skill rehabilitation per se, serious gaming metrics can reflect cognitive factors, such as attention, working memory, and decision-making processes. Although such cognitive factors are not typically regarded as relevant for understanding motor performance, cognitive abilities represent fundamental constraints on learning and execution of movements $(37,38)$.

Study limitations. This study has a couple of notable limitations. Firstly, the small number of participants was the most important major limitation of this pilot study. Studies with larger cohorts are required to validate our preliminary results and allow clinical trials focused on new rehabilitation approaches. Secondly, the applicability of the MR_CHIROD v3 is limited by the simplicity of its grip functions and the lack of multiple degrees of freedom. Notwithstanding, the device's grip functions are critical for stroke patients given that, from a neurology point of view, grip rehabilitation is the most important goal for achieving independence in daily life, including the ability to reliability grasp and hold onto objects without dropping them. In this context, it is important to note that although robotic therapy has been shown to improve arm motor function after stroke (39-43), apart from a few reports (43-45), these efforts have not focused on the hand (46) as the presently examined system does.

Conclusion. The present results demonstrate that MR CHIROD v3 device output parameters and linked serious game score elements can be used as indices of hand motor function during post-stroke hand rehabilitation training. These robot and game metrics showed especially marked improvements during the initial 2 weeks of rehabilitative training in the present study sample. Clinical motor scale scores were found to associate very strongly with these indices, with the best correlated metric being average force. Game score elements and device output parameters can be combined to build a more reliable predictive model of clinical motor performance and, potentially, replace commonly used unreliable self-assessment methods. Larger studies are needed to inform the development of models that can be used reliably for remote monitoring and patient prognosis applications.

\section{Acknowledgements}

We wish to thank Dr Bruce R. Rosen, M.D., Ph.D. (Director of the Athinoula A. Martinos Center for Biomedical Imaging), for his support in the implementation of the imaging studies.

\section{Funding}

This work was supported by a grant from the National Institute of Neurological Disorders and Stroke (grant no. 1R01NS105875-01A1) of the National Institutes of Health.

\section{Availability of data and materials}

The datasets used and/or analyzed during the current study are available from the corresponding author on reasonable request.

\section{Authors' contributions}

LGA analyzed the data and prepared the manuscript. GDN designed and created the computer game. MPO designed, 
manufactured and tested the robotic device and edited the manuscript. CP and SL acquired the raw data and assessed their authenticity. MAM provided data interpretation and manuscript editing. AAT designed and supervised the study. All authors read and approved the final manuscript.

\section{Ethics approval and consent to participate}

Institutional review board approval of the study was granted by the Partners Human Research Committee (protocol no. 2005P000570) and all participants provided written informed consent.

\section{Patient consent for publication}

Not applicable.

\section{Competing interests}

The authors declare that they have no competing interests.

\section{References}

1. Virani SS, Alonso A, Benjamin EJ, Bittencourt MS, Callaway CW, Carson AP, Chamberlain AM, Chang AR, Cheng S Delling F, et al: Heart disease and stroke statistics-2020 update: A report from the American Heart Association. Circulation 141: e139-e596, 2020

2. Centers for Disease $\mathrm{C}$ and Prevention (CDC): Public health and aging: Hospitalizations for stroke among adults aged $>/=65$ years-United States, 2000. MMWR Morb Mortal Wkly Rep 52: 586-589, 2003.

3. Indredavik B, Slordahl SA, Bakke F, Rokseth R and Haheim LL: Stroke unit treatment. Long-term effects. Stroke 28: 1861-1866, 1997.

4. Kwakkel G, Kollen B and Lindeman E: Understanding the pattern of functional recovery after stroke: Facts and theories Restor Neurol Neurosci 22: 281-299, 2004

5. Ballester BR, Maier M, Duff A, Cameirão M, Bermúdez S, Duarte E, Cuxart A, Rodríguez S, San Segundo Mozo RM and Verschure PFMJ: A critical time window for recovery extends beyond one-year post-stroke. J Neurophysiol 122: 350-357, 2019.

6. Dobkin BH and Dorsch A: New evidence for therapies in stroke rehabilitation. Curr Atheroscler Rep 15: 331, 2013.

7. Friedrich R, Hiesel P, Peters S, Siewiorek DP, Smailagic A and Brugge B: Serious games for home-based stroke rehabilitation. Stud Health Technol Inform 213: 157-160, 2015.

8. Tamayo-Serrano P, Garbaya S and Blazevic P: Gamified in-home rehabilitation for stroke survivors: Analytical review. Int J Ser Games 5: 2018 .

9. Fazekas $\mathrm{G}$ and Tavaszi I: The future role of robots in neuro-rehabilitation. Expert Rev Neurother 19: 471-473, 2019.

10. Lee HS, Park YJ and Park SW: The effects of virtual reality training on function in chronic stroke patients: A systematic review and meta-analysis. Biomed Res Int 2019: 7595639, 2019.

11. Chang WH and Kim YH: Robot-assisted therapy in stroke rehabilitation. J Stroke 15: 174-181, 2013.

12. Cho KH, Hong MR and Song WK: Upper limb robotic rehabilitation for chronic stroke survivors: A single-group preliminary study. J Phys Ther Sci 30: 580-583, 2018.

13. Oldfield RC: The assessment and analysis of handedness: The Edinburgh inventory. Neuropsychologia 9: 97-113, 1971.

14. Fugl-Meyer AR, Jääskö L, Leyman I, Olsson S and Steglind S: The post-stroke hemiplegic patient. 1. a method for evaluation of physical performance. Scand J Rehabil Med 7: 13-31, 1975.

15. Lyle RC: A performance test for assessment of upper limb function in physical rehabilitation treatment and research. In J Rehabil Res 4: 483-492, 1981.

16. Ansari NN, Naghdi S, Arab TK and Jalaie S: The interrater and intrarater reliability of the Modified Ashworth Scale in the assessment of muscle spasticity: Limb and muscle group effect. NeuroRehabilitation 23: 231-237, 2008
17. Mathiowetz V, Volland G, Kashman N and Weber K: Adult norms for the Box and Block Test of manual dexterity. Am J Occup Ther 39: 386-391, 1985.

18. Ottensmeyer MP, Li S, De Novi G and Tzika AA: Functional MRI in conjunction with a novel MRI-compatible hand-induced robotic device to evaluate rehabilitation of individuals recovering from hand grip deficits. J Vis Exp: 10.3791/59420, 2019.

19. Khanicheh A, Mintzopoulos D, Weinberg B, Tzika AA and Mavroidis C: MR_CHIROD v.2: Magnetic resonance compatible smart hand rehabilitation device for brain imaging. IEEE Trans Neural Syst Rehabil Eng 16: 91-98, 2008

20. Khanicheh A, Muto A, Triantafyllou C, Weinberg B, Astrakas L, Tzika A and Mavroidis C: fMRI-compatible rehabilitation hand device. J Neuroeng Rehabil 3: 24, 2006.

21. Woytowicz EJ, Rietschel JC, Goodman RN, Conroy SS Sorkin JD, Whitall J and McCombe Waller S: Determining levels of upper extremity movement impairment by applying a cluster analysis to the fugl-meyer assessment of the upper extremity in chronic stroke. Arch Phys Med Rehabil 98: 456-462, 2017.

22. Page SJ, Fulk GD and Boyne P: Clinically important differences for the upper-extremity Fugl-Meyer Scale in people with minimal to moderate impairment due to chronic stroke. Phys Ther 92: 791-798, 2012.

23. Chen HM, Chen CC, Hsueh IP, Huang SL and Hsieh CL: Test-retest reproducibility and smallest real difference of 5 hand function tests in patients with stroke. Neurorehabil Neural Repair 23: 435-440, 2009.

24. Comani S, Velluto L, Schinaia L, Cerroni G, Serio A, Buzzelli S, Sorbi S and Guarnieri B: Monitoring neuro-motor recovery from stroke with high-resolution EEG, robotics and virtual reality: A proof of concept. IEEE Trans Neural Syst Rehabil Eng 23: 1106-1116, 2015

25. Clark WE, Sivan M and O'Connor RJ: Evaluating the use of robotic and virtual reality rehabilitation technologies to improve function in stroke survivors: A narrative review. J Rehabil Assist Technol Eng 6: 2055668319863557, 2019.

26. Mubin O, Alnajjar F, Jishtu N, Alsinglawi B and Al Mahmud A: Exoskeletons with virtual reality, augmented reality, and gamification for stroke patients' rehabilitation: Systematic review. JMIR Rehabil Assist Technols 6: e12010, 2019.

27. Byl NN, Abrams GM, Pitsch E, Fedulow I, Kim H, Simkins M, Nagarajan S and Rosen J: Chronic stroke survivors achieve comparable outcomes following virtual task specific repetitive training guided by a wearable robotic orthosis (UL-EXO7) and actual task specific repetitive training guided by a physical therapist. J Hand Ther 26: 343-352; quiz 352, 2013.

28. Klamroth-Marganska V, Blanco J, Campen K, Curt A, Dietz V, Ettlin T, Felder M, Fellinghauer B, Guidali M, Kollmar A, et al: Three-dimensional, task-specific robot therapy of the arm after stroke: A multicentre, parallel-group randomised trial. Lancet Neurol 13: 159-166, 2014.

29. Saposnik G, Teasell R, Mamdani M, Hall J, Mcllroy W, Cheung D, Thorpe KE, Cohen LG and Bayley M; Stroke Outcome Research Canada (SORCan) Working Group: Effectiveness of virtual reality using Wii gaming technology in stroke rehabilitation: A pilot randomized clinical trial and proof of principle. Stroke 41: 1477-1484, 2010.

30. Heiss WD: Contribution of neuro-imaging for prediction of functional recovery after ischemic stroke. Cerebrovasc Dis 44: 266-276, 2017

31. Kwah LK and Herbert RD: Prediction of walking and arm recovery after stroke: A critical review. Brain Sci 6: 53, 2016.

32. Noveletto F, Hounsell MDS, Soares AV, Eichinger FLF, Sagawa Y and Bertemes Filho P: Stronger: A serious game framework for post-stroke rehabilitation. Ann Phys Rehabil Med 61: e487, 2018.

33. Oña ED, Cano-de la Cuerda R, Sánchez-Herrera P, Balaguer C and Jardón A: A review of robotics in neurorehabilitation: Towards an automated process for upper limb. J Healthc Eng 2018: 9758939, 2018

34. Bosecker C, Dipietro L, Volpe B and Krebs HI: Kinematic robot-based evaluation scales and clinical counterparts to measure upper limb motor performance in patients with chronic stroke. Neurorehabil Neural Repair 24: 62-69, 2010.

35. Chu CY and Patterson RM: Soft robotic devices for hand rehabilitation and assistance: A narrative review. J Neuroeng Rehabil 15: 9, 2018.

36. Hartwig V, Carbonaro N, Tognetti A and Vanello N: Systematic review of fMRI compatible devices: Design and testing criteria. Ann Biomed Eng 45: 1819-1835, 2017. 
37. Gallivan JP, Chapman CS, Wolpert DM and Flanagan JR: Decision-making in sensorimotor control. Nat Rev Neurosci 19: 519-534, 2018.

38. Song $\mathrm{JH}$ : The role of attention in motor control and learning. Curr Opin Psychol 29: 261-265, 2019.

39. Jack D, Boian R, Merians AS, Tremaine M, Burdea GC, Adamovich SV, Recce M and Poizner H: Virtual reality-enhanced stroke rehabilitation. IEEE Trans Neural Syst Rehabil Eng 9: 308-318, 2001.

40. Hesse S, Schulte-Tigges G, Konrad M, Bardeleben A and Werner C: Robot-assisted arm trainer for the passive and active practice of bilateral forearm and wrist movements in hemiparetic subjects. Arch Phys Med Rehabil 84: 915-920, 2003.

41. Huang VS and Krakauer JW: Robotic neurorehabilitation: A computational motor learning perspective. J Neuroeng Rehabil 6: 5, 2009.

42. Pignolo L: Robotics in neuro-rehabilitation. J Rehabil Med 41: 955-960, 2009
43. Duret $\mathrm{C}$ and Hutin E: Effects of prolonged robot-assisted training on upper limb motor recovery in subacute stroke. NeuroRehabilitation 33: 41-48, 2013.

44. Balasubramanian S, Klein J and Burdet E: Robot-assisted rehabilitation of hand function. Curr Opin Neurol 23: 661-670, 2010.

45. Metzger JC, Lambercy O, Califfi A, Dinacci D, Petrillo C, Rossi P, Conti FM and Gassert R: Assessment-driven selection and adaptation of exercise difficulty in robot-assisted therapy: A pilot study with a hand rehabilitation robot. J Neuroeng Rehabil 11: 154, 2014.

46. Prange GB, Jannink MJ, Groothuis-Oudshoorn CG, Hermens HJ and Ijzerman MJ: Systematic review of the effect of robot-aided therapy on recovery of the hemiparetic arm after stroke. J Rehabil Res Dev 43: 171-184, 2006.

(i) (9) This work is licensed under a Creative Commons Attribution-NonCommercial-NoDerivatives 4.0 International (CC BY-NC-ND 4.0) License. 